\title{
The SIRS Model of Epidemic Spreading in Virtual Society
}

\author{
A. GRABOWSKI ${ }^{a, *}$ AND R. KosińsKI ${ }^{a, b}$ \\ ${ }^{a}$ Central Institute for Labor Protection - National Research Institute \\ Czerniakowska 16, 00-701 Warsaw, Poland \\ ${ }^{b}$ Faculty of Physics, Warsaw University of Technology \\ Koszykowa 75, 00-662 Warsaw, Poland \\ Dedicated to Professor Dietrich Stauffer on the occasion of his 65th birthday
}

The phenomenon of epidemic spreading in a real social network is described and investigated numerically. On the basis of data concerning amount of time devoted daily to social interactions, the influence of human activity on spreading process is investigated in the frame of SIRS model. It was found that the activity of an individual is positively correlated with its connectivity and the relation has power law form. The influence of control measures on the spreading process is investigated as a function of initial conditions. The cost-effectiveness of mass immunizations campaigns and target vaccinations is compared. It was found that the form of activity distribution has significant influence on the spreading phenomena in the network.

PACS numbers: 89.75.-k, 89.65.--s, 87.10.--e

\section{Introduction}

In recent years it was discovered that a structure of different biological, technical, economical and social systems has the properties of complex networks [1]. The short length of the average shortest-path distance and the high value of the clustering coefficient are some of the common properties of those networks. Social networks, which are an important example of complex networks, also have those properties. They are successfully modeled using different approaches $[2,3]$, in particular, small-world topology of interpersonal connections, and their hierarchical structure are taken into account, e.g. epidemic spreading in a population with a three-level structure of interpersonal interactions was analyzed in Ref. [4]. Such a structure of a social network has a strong influence on dynamical phenomena in a population.

In recent years the spreading of epidemics was investigated by many authors, who used different models of interpersonal interactions [5-7]. Different approaches

*corresponding author; e-mail: angra@ciop.pl 
to generation of graphs with desirable properties, e.g., a degree distribution or correlations between nodes connectivity, were used [2].

Human activity has essential influence on the dynamical phenomena (e.g. epidemic spreading, opinion formation) in social networks [8]. In the present work we use data on real social network consisting of $10^{4}$ individuals, who interact in a large virtual world of Massive Multiplayer Online Role Playing Game (MMORPG) [9]. This network has similar properties to other social networks. It should be stressed that social interactions with other players are very important part of each MMORPG. Moreover, on-line games, like MMORPGs, offer a great opportunity to investigate human behavior, because much information about individuals is registered in databases. On the basis of the playing time received from the on-line game server, we observe human dynamics [10]. We calculate the activity $A$ of individuals, i.e. the relative time daily devoted to interactions with others. It was often assumed in models of epidemic spreading that intensity of interactions with others is the same for each individual. Our research has shown that distribution of activity is not uniform and is highly correlated with node degree. The aim of this work is to investigate process of the epidemic spreading (on the basis of susceptible infected removed susceptible SIRS model) in the real social network, taking into account human activity.

\section{The structure of the network and human activity}

The population consists of $N=6065$ individuals. The network under investigation is a Giant Component of the network described in Ref. [9]. A high value of the clustering coefficient $C=0.1$ and a short average path length $\langle l\rangle=4.8$ are characteristic features of social networks [2]; they are typical of small-world networks [11]. The degree distribution of the network shows power law regime $P(k) \sim\left(k+k_{0}\right)^{-\gamma}$ with $k_{0}=6$ and $\gamma=3$. Such a power law is common in many types of networks [1], also in social networks [12]. The correlation between the local clustering coefficient $C(k)$ and the node degree $k$ show the existence of a power law $C(k) \sim k^{-\alpha}$ with $\alpha=0.44$. The power-law relation $C(k)$ is similar to the relationship observed in hierarchical networks [13]. The average connectivity of the nearest neighbors $k_{\mathrm{NN}}$ of a node increases with number of its connections $k$. Hence, the network under investigation is assortative mixed by degree; such a correlation is observed in many social networks [3].

It was found that the activity distribution can be fit to an exponential form $P(A) \sim \mathrm{e}^{-12 A}$. The relation between degree of an individual and its activity shows that the greater the $k$, the greater the $A$. Hence, the activity of an individual is positively correlated with its connectivity and the results can be approximated with power law $A(k) \sim k^{0.35}$. Knowing the activity of individuals we can start to investigate the process of the epidemic spreading in the real social network. 


\section{Epidemic spreading}

In the literature there are many models of epidemic spreading with different mechanisms of contagion [4, 14]. However, in order to better understand the influence of human activity on spreading process we have used simple SIRS model $[5,15]$. In our model, each individual is in one of three permitted states: healthy and susceptible (S), ill (I), healthy and unsusceptible or isolated from the rest of the population $(\mathrm{R})$. The state of the individual evolves in time and depends on their previous state and the contacts with other individuals. The probabilities of transitions between different states in one time step are described with the following parameters: $W_{\mathrm{S} \rightarrow \mathrm{I}}$, the probability that a susceptible individual will be infected by an ill individual (this also denotes how contagious the disease is); $W_{\mathrm{I} \rightarrow \mathrm{R}}$, the probability that an ill individual will recover or be isolated from the rest of the population (e.g. in a hospital); $W_{\mathrm{R} \rightarrow \mathrm{S}}$, the probability that an unsusceptible individual loses its immunity and becomes healthy and susceptible (this value may be referred to the probability of the mutation of the pathogen).

To distinguish the effectiveness of interactions between individuals we take into account human activity $A$. We assume that the probability of an infection of an individual by one of $k$ neighbors in one time step (one day) is a simple linear function of the number of ill neighbors and has a form

$$
p_{i}=24 W_{\mathrm{S} \rightarrow \mathrm{I}} A_{i} \sum_{j}^{k_{i}^{\mathrm{I}}} A_{j},
$$

where $p_{i}$ is probability of infection per one day, $W_{\mathrm{S} \rightarrow \mathrm{I}}$ is the probability of infection per one hour of contact, $k_{i}^{\mathrm{I}}$ is the number of ill neighbors of $i$-th individual, $A_{i}$ social activity of $i$-th individual. Other probabilities of a transition between states $X, Y$ in one time step do not depend on structure of the network and human activity and they are described by the parameters $W_{X \rightarrow Y}$.

Computations were performed for the initial conditions with one ill (I) and randomly located individual and the rest of the population healthy and susceptible (S). Synchronous dynamics with assumption that an individual can change its state only one time in each time step was used. In order to investigate the dynamics of the spreading process and the range of an epidemic we introduce two observables: the time $t_{\max }$ when the maximal number of ill individuals is reached and the magnitude of epidemic $V$ defined as relative number of individuals who went through the disease during epidemic.

In order to investigate the influence of target vaccination on the process of epidemic development we introduce the parameter $p_{\mathrm{TV}}$. In each time step all $\mathrm{S}$ nearest neighbors of the ill individual, are vaccinated with the probability $p_{\mathrm{TV}}$. After vaccination, these individuals become unsusceptible (R) (to simplify the model we assume that the time necessary to develop immunity is very short no longer than one day). It should be noted that introducing the probability $p_{\mathrm{TV}}$ can be also treated as a simple model of chemoprophylaxis [16]. The value 
of the probability $p_{\mathrm{TV}}$ is related to the time of identification of ill individuals in population by health services.

The relation between control parameters describing a disease and observables ( $V$ and $t_{\max }$ ) is shown in Fig. 1. In order to investigate the influence of the human activity on the spreading process we have made computations for two different distributions of activity, real and uniform $A_{i}=$ const. The average activity was the same in both distributions, with the aim of obtaining better comparable results.
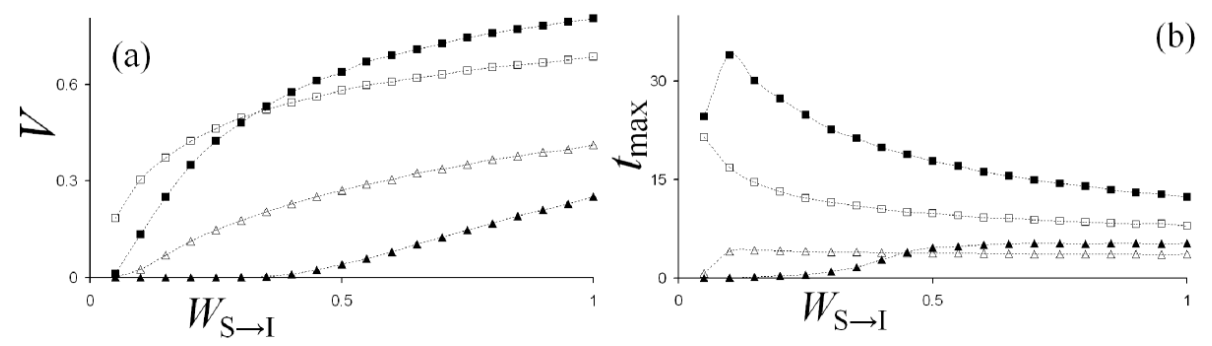

Fig. 1. The influence of the parameter $W_{\mathrm{S} \rightarrow \mathrm{I}}$ on the magnitude of epidemic $V$ (a) and time $t_{\max }(\mathrm{b})$ for different values of $W_{\mathrm{I} \rightarrow \mathrm{R}}(0.1$ - boxes and 0.9 - triangles). Black and white marks correspond to uniform and real distribution of activity, respectively. Results were averaged over $10^{4}$ independent simulations. The values of the other parameters are: $W_{\mathrm{R} \rightarrow \mathrm{S}}=0$.

For large values of $W_{\mathrm{I} \rightarrow \mathrm{R}}$ the magnitude of epidemic $V$ increases with an increase in $W_{\mathrm{S} \rightarrow \mathrm{I}}$. In the case of real distribution of activity $V$ is much larger and the value of the time $t_{\max }$ is lower. Hence, epidemic spreads faster and reaches larger part of the network. It is a result of the presence of very active super-spreaders [17], i.e. individuals with large connectivity and high value of social activity $A$. The process of epidemic is highly influenced by super-spreaders, because the probability that they are connected is large (the network is assortative mixed by degree). These individuals cause that epidemic reaches distant part of the network very fast, even in the case of low contagious diseases. In the case of uniform distribution of activity super-spreaders are less effective, because their social activity $A$ is smaller (the activity of a node is positively correlated with its degree). Therefore the epidemic cannot spread in the network (the magnitude of epidemic is close to zero for low values of $W_{\mathrm{S} \rightarrow \mathrm{I}}$ ).

For low values of $W_{\mathrm{I} \rightarrow \mathrm{R}}$ the decrease in time $t_{\max }$ is visible, hence the epidemic spreads faster with an increase in $W_{\mathrm{S} \rightarrow \mathrm{I}}$ (see Fig. 1b). The magnitude of epidemic remains large, even in the case of low contagious diseases. As a result of the presence of more active super-spreaders in the case of real distribution of connectivity, the magnitude of epidemic is larger.

However, for large values of the $W_{\mathrm{S} \rightarrow \mathrm{I}}$ opposite situation is true if the magnitude of epidemic is larger in the case of uniform distribution of activity. On 
one hand, the social activity of an individual with small number of connections is low, therefore the probability that such an individual will be infected is very low. On the other hand, the hubs, which are highly interconnected (the network is positively correlated by degree) and very active, quickly spread the disease to the other hubs. Thus, the epidemic spreads faster (see Fig. 1b), but quickly dies out. As a result, the probability that an individual with low $k$ and low $A$ will be infected during the epidemic is low. Therefore, the magnitude of epidemic is lower in the case of real distribution of activity, because the number of individuals with low $k$ and $A$ is large.

In order to investigate the influence of routine preventive vaccination on the spreading process, at the time $t=0$ the state of $N_{\mathrm{R} 0}$ randomly chosen individuals is set to $(\mathrm{R})$. With an increase in the number of preventively vaccinated individuals $N_{\mathrm{R} 0}$, there is a decrease in the magnitude of epidemic (Fig. 2). This is so because an epidemic cannot spread freely in the presence of vaccinated individuals. In the case $W_{\mathrm{R} \rightarrow \mathrm{S}}>0$ the behavior of the system is more complicated. There is a non-zero probability $P_{\mathrm{E}}$ of occurrence of an endemic state (we define $P_{\mathrm{E}}$ as a probability that after $10^{4}$ time steps the number of ill or infected individuals is greater than zero). In the case of real distribution of activity the presence of very active super-spreaders causes an increase in probability $P_{\mathrm{E}}$. Moreover, the value of the probability $P_{\mathrm{E}}$ decreases much slower with an increase in $N_{\mathrm{R} 0}$. Therefore the magnitude of an epidemic increases abruptly with an increase in $W_{\mathrm{R} \rightarrow \mathrm{S}}$, and $V$ is relatively large even if almost whole population was vaccinated. It indicates that mass routine vaccination is not optimal in the case of easily mutating pathogens.

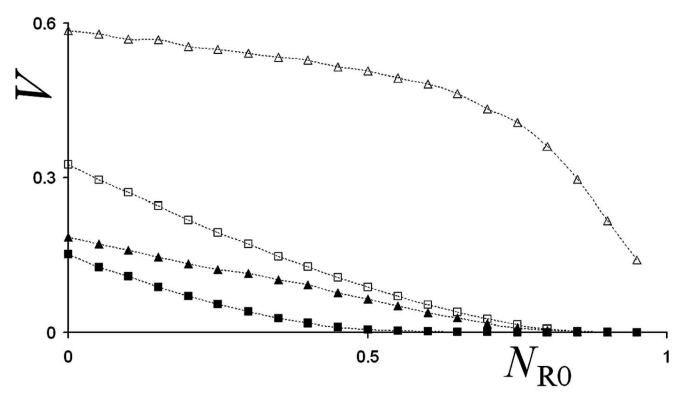

Fig. 2. The influence of the number of preventive vaccinated individuals $N_{\mathrm{R} 0}$ on the magnitude of epidemic $V$ for different values of $W_{\mathrm{R} \rightarrow \mathrm{S}}(0-$ boxes and 0.0015 - triangles). Black and white marks correspond to uniform and real distribution of activity, respectively. Results were averaged over $10^{4}$ independent simulations. The values of the other parameters are: $W_{\mathrm{S} \rightarrow \mathrm{I}}=0.3, W_{\mathrm{I} \rightarrow \mathrm{R}}=0.3$.

The routine preventive vaccination is not the only method of using vaccines. In our work, we also investigated the influence of target vaccination [18]. Figure 3 illustrates the influence of the probability $p_{\mathrm{TV}}$ on the spreading process (in the simulation we assume that there was no shortage of vaccines). The magnitude 
of epidemic decreases with an increase in $p_{\mathrm{TV}}$. It is visible that in the case of real distribution of activity the value of $V$ is greater. Moreover, it decreases much slower with an increase in $p_{\mathrm{TV}}$ than in the case of uniform distribution of connectivity. The difference in results for different distributions of activity is better visible for larger values of the parameter $W_{\mathrm{R} \rightarrow \mathrm{S}}$.
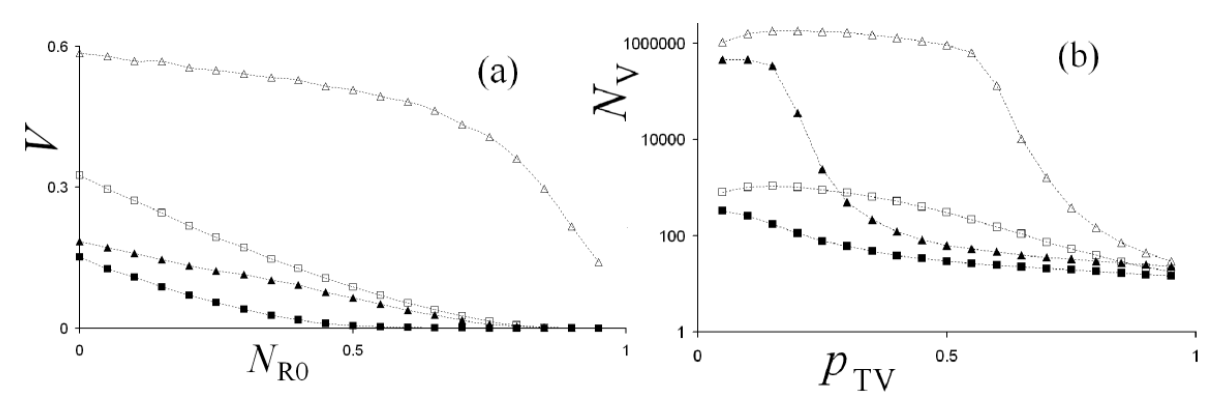

Fig. 3. The influence of the probability $p_{\mathrm{TV}}$ on the magnitude of the epidemic $V$ (a) and the number of vaccinated individuals $N_{\mathrm{V}}$ (b) for different values of $W_{\mathrm{R} \rightarrow \mathrm{S}}$ ( 0 - boxes and 0.25 - triangles). Black and white marks correspond to uniform and real distribution of activity, respectively. Results were averaged over $10^{4}$ independent simulations. The values of the other parameters are: $W_{\mathrm{S} \rightarrow \mathrm{I}}=0.3, W_{\mathrm{I} \rightarrow \mathrm{R}}=0.3$.

Because of the cost of vaccines, it is important to calculate the number $N_{\mathrm{V}}$ of individuals who are vaccinated (Fig. 3b). Although in our model we assume unlimited supplies of vaccines, during a real epidemic a shortage of vaccines is quite likely. The value of $N_{\mathrm{V}}$ decreases with an increase in $p_{\mathrm{TV}}$ and is greater in the case of real distribution of activity. When the new ill individuals are identified quickly enough (large values of $p_{\mathrm{TV}}$ ) an epidemic can be suppressed with relatively small numbers of vaccines, even if the probability that an individual loses its immunity is large. In our simulations we assumed that in time $t=0$ only one individual is infected. In the case when the number of initially ill individuals is large (e.g. as result of broad dispersal of pathogens during bio-terrorist attack) even very quick identification of new ill individuals $\left(p_{\mathrm{TV}} \approx 1\right)$ is insufficient: the magnitude of the epidemic and the number of vaccines used remain relatively large [18].

\section{Conclusions}

We have shown that human activity has significant influence on dynamic processes in social network. It was found that the activity $A$ of an individual (i.e. the relative time daily devoted to interactions with others) is positively correlated with its degree. The process of an epidemic spreading in a real social network has been investigated numerically. It occurs that in the case of real distribution of activity the epidemic spreads faster and for a large range of values of the control parameters the magnitude of epidemic is larger. This is a result of the presence of 
very active super-spreaders (individuals with high degree and large value of social activity) in the social network. It should be stressed that in the case of an epidemic in a real population the pattern of human behavior will change. In time of severe epidemic people decrease the time devoted daily to interactions with others (social activity $A$ ) in order to avoid an infection. However, we suggest that in the case of limited epidemics (low values of $W_{\mathrm{S} \rightarrow \mathrm{I}}$ ), when the number of ill individuals is relatively low (e.g. annual influenza epidemics), the change in social activity is small, too. Therefore, we suggest that the models taking into account data on social activity of humans presented in this work seem to be more plausible for modeling the process of epidemic spreading in human population than previous models presented in literature.

In our model, the influence of routine preventive vaccinations on the spreading of an epidemic was investigated. We found that the use of routine preventive vaccinations can suppress an epidemic. However, the vaccine coverage is very high.

Contrary to routine preventive vaccination, target vaccination can give much better results with little demand for vaccines (or antiviral agents), i.e. when only the nearest neighbors of ill individuals are vaccinated. An epidemic can be suppressed with a relatively small number of vaccines if new ill individuals are identified quickly enough. However, it is not easy to identify super-spreaders in the community.

Routine preventive vaccination can be effective only in the case of well known pathogens (e.g. in the case of childhood diseases such as measles). If a new pathogen appears in a susceptible population (as a result of mutation or a bioterrorist attack), only a quick public health response can provide good results. In such case, the efficiency of target vaccination of the nearest neighbors of ill individuals is high.

Our model provides an opportunity to study the influence of routine preventive vaccination as well as targeted vaccinations on the spread of an epidemic, taking into account real distribution of human activity coming from on-line community. This is of particular interest since these measures are frequently implemented in practice.

\section{Acknowledgments}

A.G. acknowledges financial support from the Foundation for Polish Science (FNP 2008).

\section{References}

[1] R. Albert, A.-L. Barabási, Rev. Mod. Phys. 74, 47 (2002).

[2] S.N. Dorogovtsev, J.F.F. Mendes, Evolution of Networks, Oxford Univ. Press, Oxford 2004.

[3] M.E.J. Newman, Phys. Rev. E 67, 026126 (2003).

[4] A. Grabowski, R. Kosiński, Phys. Rev. E 70, 031908 (2004). 
[5] N.T.J. Bailey, The Mathematical Theory of Infectious Diseases, Griffin, London 1975.

[6] S.M. Dammer, H. Hinrichsen, Phys. Rev. E 68, 016114 (2003).

[7] M. Boguna, R. Pastor-Satorras, Phys. Rev. E 66, 047104 (2002).

[8] A. Vazquez, B. Rácz, A. Lukács, A.-L. Barabási, Phys. Rev. Lett. 98, 158702 (2007).

[9] A. Grabowski, N. Kruszewska, Int. J. Mod. Phys. C 18, 1527 (2007).

[10] A.-L. Barabási, Nature 435, 207 (2005).

[11] S.H. Strogatz, Nature (London) 410, 268 (2001).

[12] F. Liljeros, C.R. Edling, L.A.N. Amaral, H.E. Stanley, Y. Aberg, Nature 411, 907 (2001).

[13] E. Ravasz, A.L. Barabási, Phys. Rev. E 67, 026112 (2003).

[14] D. Centola, V.M. Eguiluz, M.W. Macy, Physica A 374, 449 (2007).

[15] W.O. Kermack, A.G. McKendrick, Proc. R. Soc. Lond. A 115, 700 (1927).

[16] I.M. Longini, M.E. Halloran, A. Nizam, Y. Yang, Am. J. Epidemiol. 159, 623 (2004).

[17] M. Barthelemy, A. Barrat, R. Pastor-Satorras, A. Vespignani, Phys. Rev. Lett. 92, 178701 (2004).

[18] A. Grabowski, M. Rosińska, Acta Phys. Pol. B 37, 1521 (2006). 